\title{
Reducing the silence
}

\begin{abstract}
The suicide rate in the US is increasing, whereas funding for research into suicide prevention has decreased. It will take more investment to truly understand the mechanisms of action underlying the causes of this global killer and to design new treatments for those causes. But efforts must come from all segments of society.
\end{abstract}

T he standard review process used by the US Food and Drug Administration (FDA) for prescription drugs can be slow, taking the better part of a year, but designating a drug as a 'breakthrough therapy' can accelerate the approval process. In August, one such designation was given to esketamine, a form of the more commonly known ketamine, with the indication for major depression with risk of suicide. The importance of accelerating the development of such treatments is highlighted by the fact that it is the second leading cause of death worldwide among 15-29 year olds ${ }^{1}$. Furthermore, in the US, suicide rates are at their highest in 30 years. The emotional and physical tolls of this tragedy cannot be overstated.

The FDA has approved no drugs for the indication of risk of suicide, which gives particular importance to the prospect of esketamine trials. The current best practice for at-risk individuals is to admit them to the emergency room to be placed under supervision, for what is usually a short period known as suicide watch. A societal extension of this approach is making changes to infrastructure, such as adding barriers to the edges of bridges. Of course, not all suicides can be prevented in this way, and a targeted pharmacological approach could go further to prevent more deaths and help to ameliorate helpless moods. Importantly, existing data indicate that ketamine is fast-acting and so could rapidly raise mood to prevent suicide attempts by those with suicidal ideations ${ }^{2}$. This is particularly compelling given that a mood disorder is present in at least $50 \%$ of suicides, and that currently, up to one-third of individuals with major depression do not respond to existing therapies ${ }^{3}$.

The majority of ketamine and ketamine-derivative research to date has been for major depressive disorder, and it is thought that the drug might act to prevent suicide through its immediate effect of improving mood. Yet, the lack of a more complete understanding of mechanism of action remains an urgent gap in our knowledge. Ketamine is a known blocker of the NMDA-receptor protein, and it was previously thought that the drug mediated its antidepressant effects through this pathway. However, a recent study carried out in mice and reported in Nature in April called this mechanism for mediation of mood-altering effects into question ${ }^{4}$. Further understanding of its mechanism of action could enable the development of drugs that are more specific and effective at raising mood, which could also possibly reduce side effects-ketamine can result in dissociative effects even at low doses. Frustratingly, we also know little about how to identify those who are at risk of suicide, either in terms of behavior or in terms of biological measures such as brain-circuit activity and genetics.

Studies can and will shed more light on this, but there are ethical complications to recruiting subjects who have recently attempted suicide from the emergency room, and researchers are only just beginning to navigate these. Those at risk of suicide are also still routinely excluded from clinical trials, which adds to the difficulty of monitoring the effects of pharmacological intervention on suicide. With respect to this, it is only in recently approved trials of esketamine that the indication of risk of suicide has been added.

In the US, funding for research into suicide prevention comes overwhelmingly from public resources. A report published last year by the country's National Action Alliance for Suicide Prevention, a public-private partnership, indicated that $98 \%$ of studies on suicide prevention between 2008 and 2013 were funded by the government, and that $2 \%$ came from private resources such as the American Foundation for Suicide Prevention (although it is worth noting that the threshold for inclusion of a foundation in the report was an investment of $\$ 100,000$ or more for suicide-related studies, and that other investment into underlying mental disorders was not included $)^{5}$. The report called the funding in this area "meager", both with respect to public and private investment. For context, 43,000 people in the US died as a result of suicide in 2014-approximately the same number of individuals who died of breast cancers that year $(40,000)$. Yet it is clear that the discussion around suicide prevention does not reflect these numbers.

Without further funding, we will make little progress in understanding the molecular and cellular basis of suicide ideations and will therefore be unable to develop new therapies. Efforts such as these that aim to raise societal awareness of suicide are to be commended and are part of the type of holistic approach that is necessary. Talking openly about suicide could help to reduce any lingering societal stigma and encourage stronger funding. Journals also have a role here, by publishing pieces to advocate for further funding, and by publishing new mechanistic and therapeutic insights generated by research funded as a consequence of those efforts. The current status quo of not discussing suicide must change, and in the wake of this change will come funding for research into this serious lifethreatening condition, and the first approved therapeutic for those at risk of suicide.

1. World Health Organization. Preventing Suicide (WHO, 2014).

2. Duman, R.S., Aghajanian, G.K., Sanacora, G. \& Krystal, J.H. Nat. Med. 22, 238-249 (2016).

3. Trevino, K., McClintock, S.M., Fischer, N.M., Vora, A. \& Husain, M.M. Ann. Clin. Psychiatry 26, 222-232 (2014).

4. Zanos, P. et al. Nature 533, 481-486 (2016)

5. National Action Alliance for Suicide Prevention: Research Prioritization Task Force. U.S. National Suicide Prevention Research Efforts: 2008-2013 Portfolio Analyses (National Institute of Mental Health and Research Prioritization Task Force, 2015). 